\title{
Budesonide/Formoterol Maintenance and Reliever Therapy versus Free-Combination Therapy for Asthma: A Real-Life Study
}

\author{
Erhaltungs- und Bedarfstherapie bei Asthma mit Budesonid/Formoterol im Ver- \\ gleich zur freien Kombinationstherapie: Eine Studie unter Alltagsbedingungen
}

Author

Institution

\section{P. Kardos}

Group Practice and Centre for Pneumology, Allergy and Sleep Medicine at Red Cross Maingau Hospital, Frankfurt am Main, Germany received 7.5 .2013

accepted after revision 10.6.2013

\section{Bibliography}

Dol http://dx.doi.org/ 10.1055/s-0033-1344349 Pneumologie 2013; 67: 463-470

(c) Georg Thieme Verlag KG Stuttgart · New York ISSN 0934-8387

\section{Corresponding author} Peter Kardos

Centre for Pneumology, Allergy and Sleep Medicine at Maingau Hospital Scheffelstraße 2 60318 Frankfurt am Main Germany Kardos@LungenpraxisMaingau.de

\section{Abstract \\ $\nabla$}

Background: This non-interventional real-life study (NCT00884689) compared budesonide/formoterol (Symbicort ${ }^{\circledR}$ ) maintenance and reliever therapy (SMART ${ }^{\mathrm{TM}}$ ) with a free combination of inhaled corticosteroid (ICS) + long-acting $\beta_{2}$-agonist (LABA) (in separate inhalers) and as-needed short-acting $\beta_{2}$-agonist (SABA) in adult asthma patients with an exacerbation in the past 24 months.

Methods: Asthma patients received SMART ${ }^{\mathrm{TM}}$ or free-combination ICS + LABA and as-needed SABA for 6 months. Allocation of patients and doses prescribed were at physician's discretion. No other restrictions applied. Primary endpoint: mean no. of rescue medication puffs/day.

Results: 482 patients were included (SMARTTM $\mathrm{n}=310$; free combination $\mathrm{n}=172$ ). SMART ${ }^{\mathrm{TM}}$ patients used less rescue medication vs the freecombination group (mean difference -0.27 puffs/day; $p=0.013)$. Severe asthma exacerbations were rare (mean rate 0.20 vs $0.17 /$ year for SMART $^{\mathrm{TM}}$ vs free combination). The mean ICS dose was $615 \mu \mathrm{g}$ in SMART ${ }^{\mathrm{TM}}$ and $678 \mu \mathrm{g}$ in freecombination group.

Conclusions: In this real-life setting, SMART ${ }^{\mathrm{TM}}$ treated patients used less rescue medication despite a lower ICS burden vs conventional asthma management with three different inhalers.

\section{Introduction}

\section{$\nabla$}

The Global Initiative for Asthma (GINA) guideline emphasises the combination of inhaled corticosteroids (ICSs) and long-acting $\beta_{2}$-agonists (LABAs) as the first-choice treatment in patients with asthma who are not controlled with ICS alone (GINA treatment Step 3 and higher) [1]. These treatments can be administered via two se-

\section{Zusammenfassung \\ $\nabla$}

Hintergrund: Diese nicht-interventionelle Studie (NCT00884689) verglich Budesonid/Formoterol (Symbicort ${ }^{\circledR}$ maintenance and reliever therapy, SMART ${ }^{\mathrm{TM}}$ ) mit der freien Kombination eines inhalativen Kortikosteroids (ICS) und langwirksamem Beta2-Sympathomimetikums (LABA) in separaten Inhalatoren in Verbindung mit einem kurzwirksamen Beta2-Sympathomimetikum (SABA) als Bedarfsmedikation.

Methode: Die Zuteilung von erwachsenen Patienten und die verschriebene Dosis lagen im Ermessen des Arztes. Primärer Endpunkt war die mittlere Anzahl der Hübe der Notfallmedikation pro Tag.

Ergebnisse: 482 Patienten wurden in die Studie eingeschlossen (SMART ${ }^{\mathrm{TM}} \mathrm{n}=310$; freie Kombination $\mathrm{n}=172$ ). Die SMART ${ }^{\mathrm{TM}}$ Gruppe verwendete weniger Notfallmedikation als die freie Kombinationsgruppe (mittlere Differenz - 0,27 Hübe/Tag; $\mathrm{p}=0,013$ ). Schwerwiegende Asthma-Exazerbationen waren selten (mittlere Rate 0,20 vs. $0,17 / \mathrm{Jahr}$ für SMART ${ }^{\mathrm{TM}}$ vs. freie Kombination). Die mittlere ICS-Dosis (BDP Äquivalent) betrug $615 \mu \mathrm{g}$ (SMART TM_Gruppe) und 678 $\mu \mathrm{g}$ (freie Kombination).

Schlussfolgerung: Unter Alltagsbedingungen im Vergleich zur freien Kombination verwendeten Patienten unter SMART ${ }^{\mathrm{TM}}$ Regime, trotz einer geringeren ICS-Gesamtdosis, weniger Notfallmedikation.

parate inhalers or as a fixed-dose combination via a single inhaler; the latter approach may have benefits with regard to patient adherence, if both components are clinically indicated [2]. This is particularly important in the era of uncertainty and continuous discussion about rare, potentially fatal side effects of LABA monotherapy, given that fixed-combination dosing with a single inhaler ensures concomitant delivery of ICS and LABA [3]. 
The fixed-dose combination of budesonide/formoterol (Symbicort $^{\circledR}$ Turbuhaler $^{\circledR}$, AstraZeneca) can be used as both maintenance and reliever therapy in adults [4]. This treatment strategy is known as SMART ${ }^{\mathrm{TM}}$ (Symbicort ${ }^{\circledR}$ maintenance and reliever therapy), and is recommended in doses of $160 / 4.5 \mu \mathrm{g}$ per inhalation, administered as either one or two inhalations twice daily and additional as-needed reliever doses. A series of randomised, controlled trials (RCTs) has demonstrated the clinical superiority of the SMART ${ }^{\mathrm{TM}}$ regimen over the conventional free combination of ICS and LABA with additional short-acting $\beta_{2}$-agonist (SABA) as required, via separate inhaler [5 -7]. However, the external validity of data gathered from carefully controlled RCTs in the real world is questionable [8]. Indeed, the effectiveness of the SMART $^{\mathrm{TM}}$ treatment regimen in comparison with traditional treatment (ICS, LABA and reliever from three discrete inhalers) under real-life conditions is not clear.

This non-interventional study, performed in a real-life clinic setting, therefore aimed to evaluate the use of rescue medication and health-related quality of life (HRQL) in adult patients with asthma who received budesonide/formoterol as maintenance therapy plus additional inhalations as needed to control symptoms (SMART ${ }^{\mathrm{TM}}$, single-inhaler therapy). These patients were compared with patients who received a free combination of an ICS and an inhaled LABA plus as-needed use of SABA, all via separate inhalers. The prescribed doses used in this study were at the discretion of the treating physician according to the recommendations of the package inserts of each medication.

\section{Methods}

$\nabla$

\section{Study design and patients}

Patients were recruited from the German centres involved in the EuroSMART study [9]. By collecting data from a real-life population, very few restrictions by inclusion and exclusion criteria were applied. However, to ascertain that only asthma patients with an indication for GINA Step 3 treatment were included, (historical) proof of reversible airway flow limitation and asthma exacerbation history were required. Therefore, this multicentre, non-interventional, real-life study (AstraZeneca study code: NISRDE-SYM-2009/1; ClinicalTrials.gov identifier: NCT00884689) included male and female outpatients aged $\geq 18$ years who had a diagnosis of asthma with reversible obstruction confirmed within the previous 5 years. Patients were receiving either budesonide/formoterol SMART ${ }^{\mathrm{TM}}$ therapy (single inhaler) or a free combination of ICS plus LABA with SABA rescue medication (via three separate inhalers) for $\geq 3$ months, with no change in treatment within 1 month of study entry. Patients were also required to have had a severe asthma exacerbation within the past 24 months, but not within 1 month of study entry. Severe asthma exacerbations were defined as occurrence of at least one of the following: use of systemic glucocorticosteroids due to asthma for $\geq 3$ days; unscheduled healthcare visit due to asthma; and hospitalisation and/or emergency room visit due to asthma requiring treatment with systemic glucocorticosteroids. Patients who had used oral, rectal or parenteral glucocorticosteroids within 30 days, used $\beta$-blockers or who had a respiratory infection within 30 days were excluded from participation in the study.

\section{Treatments}

Asthma medication and doses used were not assigned randomly and were administered at the discretion of the prescribing physician, according to standard medical practice, in a naturalistic study design. Treatments were obtained as commercially available medication; the sponsor did not provide any medication for this study. Investigators were provided with the package insert for budesonide/formoterol including dosage and SMART ${ }^{\text {TM }}$ treatment principles. No other restrictions were applied. No concomitant therapies were disallowed (with the exception of systemic corticosteroids and $\beta$-blockers), but investigators were asked to take into account relevant information from the budesonide/formoterol summary of product characteristics [4]. Likewise, the only direction given to investigators regarding the comparator group was that these patients had to be treated with ICS plus LABA and as-needed SABA via separate inhalers and should be treated according to the relevant information in the product package inserts. The physician had to document relevant data during a routine visit at inclusion, approximately three (visit 2) and six months (visit 3) after start of treatment.

\section{Outcomes}

The primary endpoint of this trial was the use of rescue medication, defined as the mean daily number of rescue puffs of budesonide/formoterol SMART ${ }^{\mathrm{TM}}$ (regular morning and evening maintenance treatment with budesonide/formoterol was not included) vs use of SABA in the free-combination arm. This was recorded using daily patient diaries during the 6 -month follow-up period. Secondary endpoints included: HRQL (differences from baseline and between group differences, not accounting for baseline differences); the number of, and time to, severe asthma exacerbations (defined as deterioration of asthma leading to at least one of the following: oral/systemic glucocorticosteroid treatment for $\geq 3$ days; unscheduled healthcare visit; or hospitalisation or emergency room visit requiring treatment with oral/systemic glucocorticosteroids); change in forced expiratory volume in $1 \mathrm{~s}$ $\left(\mathrm{FEV}_{1}\right)$ from Visit 1 to Visit 3; the number of, and time to, hospitalisation and/or emergency room treatments; level of patient satisfaction with treatment; and tolerability.

HRQL was assessed using the standardised version of the validated, asthma-specific Asthma Quality of Life Questionnaire [AQLQ(S)] [10]. This 32-item questionnaire is scored on a 7-point scale where 1 = severe impairment and 7 = no impairment; therefore, higher scores on the $A Q L Q(S)$ indicate more favourable HRQL. The average daily use of ICS over the entire study period was calculated in terms of beclomethasone propionate powder equivalents. The calculation also included the corticosteroids inhaled during rescue puffs in the SMART ${ }^{\mathrm{TM}}$ group; patients in the free-combination group did not use ICS for reliever inhalation. Patients were asked to rate their overall satisfaction with their current treatment by choosing one of the following options: very satisfied, fairly satisfied, satisfied, dissatisfied or very dissatisfied.

Tolerability was investigated by reporting of adverse events (AEs), serious AEs and treatment-related AEs. No clinical laboratory parameters were assessed.

Patients were followed up at 3 and 6 months. The study was managed by ClinResearch GmbH, Cologne, Germany, an independent clinical research organisation, and was funded by AstraZeneca. 


\section{Statistical methods}

The mean daily number of as-needed puffs of rescue medication (primary endpoint) was analysed using an analysis of covariance (ANCOVA) model with treatment as factor. Potential confounders in the analysis were identified as body mass index, gender, smoking status and season of the year at the individual study start. Therefore, a logistic regression model with treatment assignment as the dependent variable and the confounders found in the first step as independent factors was applied. Only 'season at study start' had a p-value below the predefined limit of 0.1. Therefore, the mean number of puffs of rescue medication per day was calculated using ANCOVA with treatment and propensity score as the factor, where the propensity score was calculated as the probability of having SMARTTM assigned as treatment given the season at study start.

The difference between the two treatment groups in the change from baseline in AQLQ(S) scores was evaluated using a t-test, which did not take baseline differences into account. To assess the impact of baseline differences, the mean change from baseline in the overall $\mathrm{AQLQ}(\mathrm{S})$ score was analysed using ANCOVA with treatment as factor and the baseline value as covariate.

It was planned in the study protocol that the time to first severe asthma exacerbation and the time to first hospitalisation/emergency room treatment would be evaluated. However, due to the small number of patients who experienced these endpoints, the planned analyses could not be performed and no robust statistical comparisons could be made.

\section{Sample size considerations}

Approximately 500 patients with asthma were planned to be included in this non-interventional study across approximately 60 sites in Germany. From a maximum of 20 patients, each site was expected to enrol two-thirds of patients who were treated according to the budesonide/formoterol SMART TM principle and one-third of patients who had been prescribed a free-combination treatment.

While all analyses were carried out for exploratory purposes, some statistical considerations concerning the sample size were provided by the protocol. A sample size of 333 patients in the SMART ${ }^{\mathrm{TM}}$ group and 167 patients in the free-combination group was predicted to have $80 \%$ power to detect a difference in the mean number of rescue medication puffs per day of 0.506 , using a two-group t-test with a two-sided significance level of $p<0.05$. A mean of 2.2 puffs per day and a common standard deviation (SD) of 1.9 were assumed based on data from a subgroup analysis of GINA treatment Step 3 and 4 patients from a previous study (DESOLO) [11]. It was estimated that even if the true enrolment was $350 / 151$ patients, there would be $80 \%$ power to detect a difference in daily use of rescue medication between the two treatment groups of $\geq 0.52$ actuations.

\section{Analysis sets}

The safety analysis set included all patients enrolled into the study; the full analysis set (FAS) was defined as all patients who had at least one efficacy assessment; and the sensitivity analysis set (SAS) consisted of all patients with none of the following protocol deviations: at least one inclusion criteria not fulfilled; date of written informed consent after date of Visit 1; patient prescribed a treatment other than Symbicort SMART ${ }^{\mathrm{TM}}$ or a free combination of an ICS plus LABA plus as-needed SABA; no severe asthma exacerbations documented in the last 24 months before
Visit 1; at least one exclusion criteria fulfilled; and the administered therapy was not the same as the prescribed therapy.

\section{Ethical considerations}

This non-interventional study was performed in accordance with the ethical principles outlined in the Declaration of Helsinki and is consistent with ICH/Good Clinical Practice, applicable regulatory requirements in Germany and AstraZeneca policy. All patients provided written, informed consent prior to study inclusion. The Ethics Committee of the Hessen Medical Association (Landesärztekammer Hessen) also agreed on the protocol.

\section{Results \\ $\nabla$}

Patients

The flow of patients through this study is shown in $\square$ Fig. 1. The first patient was enrolled into the study on 21 April 2009 and the last patient's final visit was 02 June 2010. A total of 498 patients were enrolled at 49 sites in Germany; of these, 482 patients were included in the FAS. Patients' demographic and clinical characteristics were similar between the SMART ${ }^{\mathrm{TM}}$ and free-combination treatment groups ( $\bullet$ Table 1 ). However, patients in the SMART ${ }^{\mathrm{TM}}$ group were more likely to be very satisfied with their current treatment (39.0\% vs $23.3 \%$ ) than free-combination treatment recipients.

$273(88.1 \%)$ of the patients in the SMARTTM group had been receiving Symbicort ${ }^{\circledR}$ for more than 6 months before study entry; $152(88.4 \%)$ of the patients in the free-combination group had been taking the ICS product they received during the study for 6 months before entering the study.

Overall, patients had high mean \pm SD AQLQ(S) scores $(5.37 \pm 1.02$ and $4.89 \pm 1.19$ points in the SMART ${ }^{\mathrm{TM}}$ and free-combination group, respectively) and high levels of treatment satisfaction: $98.1 \%$ of the SMART TM and $89.0 \%$ of the free-combination group were very satisfied, fairly satisfied or satisfied with treatment.

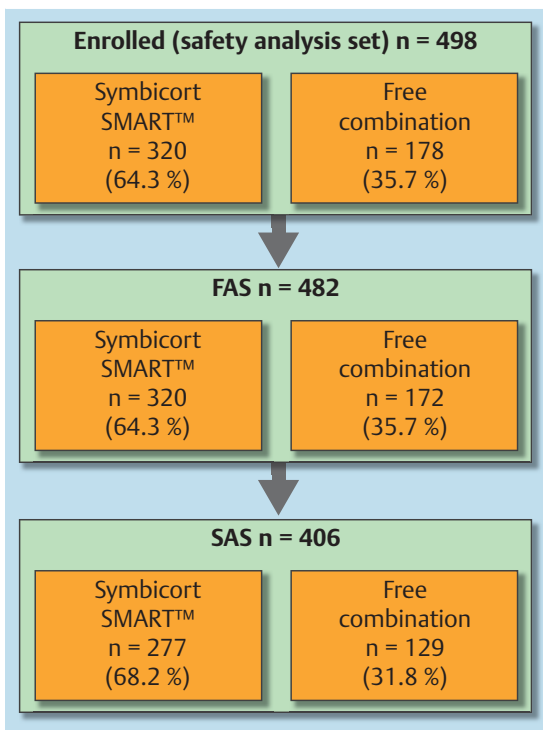

Fig. 1 Flow of patients who received SMART ${ }^{\mathrm{M}}$ or a free combination of ICS plus LABA and asneeded SABA through the study. The safety analysis set consisted of all patients enrolled in the study; the FAS was defined as all enrolled patients who had at least one efficacy assessment. The SAS included patients with no protocol deviations.

Abbreviations: FAS, full analysis set; ICS, inhaled corticosteroid; LABA long-acting $\beta_{2}$-agonist; SABA, short-acting $\beta_{2}$-agonist; SAS, sensitivity analysis set; SMART ${ }^{\mathrm{TM}}$, Symbicort ${ }^{\circledR}$ [budesonide/formoterol] maintenance and reliever therapy. 
Table 1 Baseline demographic and clinical characteristics of patients who received SMART ${ }^{T M}$ or a free combination of ICS plus LABA and as-needed SABA (full analysis set).

\begin{tabular}{|c|c|c|}
\hline Characteristics & $\begin{array}{l}\text { SMART'M } \\
(\mathrm{n}=310)\end{array}$ & $\begin{array}{l}\text { Free combination } \\
(n=172)\end{array}$ \\
\hline Age, years & $49.1(15.2)$ & $51.4(15.4)$ \\
\hline Male, $n(\%)$ & $119(38.4)$ & $59(34.3)$ \\
\hline \multicolumn{3}{|l|}{ Smoking status, $n(\%)$ : } \\
\hline Current smokers & $31(10.0)$ & $18(10.5)$ \\
\hline Ex-smokers & $63(20.3)$ & $37(21.5)$ \\
\hline Never-smokers & $216(69.7)$ & $117(68.0)$ \\
\hline \multicolumn{3}{|l|}{ Pulmonary function } \\
\hline $\mathrm{FEV}_{1}, \mathrm{I}$ & $2.64(0.87)$ & $2.45(0.73)$ \\
\hline $\mathrm{PEF}, \mathrm{I} / \mathrm{s}$ & $6.16(2.16)$ & $5.65(2.08)$ \\
\hline VC, I & $3.45(1.02)$ & $3.33(0.91)$ \\
\hline $\begin{array}{l}\text { Severe asthma exacerbations } \\
\text { within } 24 \text { months }\end{array}$ & $1.4(0.7)$ & $1.7(1.5)$ \\
\hline \multicolumn{3}{|l|}{ Asthma treatment Step, $\mathrm{n}(\%):^{1}$} \\
\hline GINA Step 3 & $238(76.8)$ & $138(80.2)$ \\
\hline GINA Step 4 & $72(23.2)$ & $34(19.8)$ \\
\hline Comorbid allergy, $\mathrm{n}(\%)^{2}$ & $27(8.7)$ & $13(7.6)$ \\
\hline AQLQ(S) global score & $5.37(1.02)$ & $4.89(1.19)$ \\
\hline \multicolumn{3}{|l|}{ Therapy satisfaction, $\mathrm{n}(\%)$ : } \\
\hline Very satisfied & $121(39.0)$ & $40(23.3)$ \\
\hline Fairly satisfied & $94(30.3)$ & $55(32.0)$ \\
\hline Satisfied & $89(28.7)$ & $58(33.7)$ \\
\hline Unsatisfied & $6(1.9)$ & $19(11.0)$ \\
\hline Prior medication, $\mathrm{n}(\%)^{3}$ & $35(11.3)$ & $7(4.1)$ \\
\hline \multicolumn{3}{|l|}{ Concomitant medication, $\mathrm{n}(\%)^{3}$} \\
\hline Systemic corticosteroids & $13(4.2)$ & $5(2.9)$ \\
\hline Systemic antibacterials & $5(1.6)$ & $0(0.0)$ \\
\hline Cough and cold preparations & $5(1.6)$ & $0(0.0)$ \\
\hline Systemic antihistamines & $0(0.0)$ & $1(0.6)$ \\
\hline
\end{tabular}

Values presented are mean (SD), unless otherwise stated.

Abbreviations: AQLQ(S), standardised version of the Asthma Quality of Life Questionnaire; $\mathrm{FEV}_{1}$, forced expiratory volume in $1 \mathrm{~s}$; GINA, Global Initiative for Asthma; ICS, inhaled corticosteroid; LABA, long-acting $\beta_{2}$-agonist; PEF, peak expiratory flow; SABA, short-acting $\beta_{2}$-agonist; SD, standard deviation; SMART ${ }^{\mathrm{M}}$, Symbicort ${ }^{\circledR}$ [budesonide/ formoterol] maintenance and reliever therapy; VC, vital capacity.

${ }^{1}$ Although all included patients had to be on ICS + LABA, investigators were asked to assess asthma severity retrospectively (i. e. before treatment was introduced).

${ }^{2}$ Allergy included: seasonal allergy, multiple allergies, hypersensitivity and house dust allergy.

${ }^{3}$ Prior medication was defined as medication that was discontinued on the calendar day prior to the first study visit, or earlier; only drugs for asthma treatment were recorded.

\section{Use of rescue medication (primary endpoint)}

In the FAS, the least squares mean daily number of rescue medication puffs (primary endpoint) was 0.557 in SMART TMtreated patients and 0.822 in free-combination recipients. This represents a difference of -0.266 (95\% confidence interval $[\mathrm{Cl}]-0.474,-0.057)$ puffs per day in favour of SMARTTM $(p=0.013)$. The difference between the treatment groups in the SAS was -0.209 (95\% CI $-0.414,-0.004)$, again favouring $\operatorname{SMART}^{\mathrm{TM}}(\mathrm{p}=0.046)$. The unadjusted mean daily number of rescue medication puffs was 0.548 for SMART TM and 0.839 for the free-combination cohort.

\section{AQLQ(S)}

The mean \pm SD AQLQ(S) global score was higher in the SMARTTM group than in the free-combination treatment group at study end ( $5.60 \pm 0.99$ vs $5.29 \pm 1.15$ points). However, the mean AQLQ(S) global score change from baseline to study end (in-group difference) was greater in the free-combination group than in SMART TM recipients ( $\bullet$ Table 2 ).

Improvements in the $\mathrm{AQLQ}(\mathrm{S})$ domain scores for symptoms, activity limitation, emotional function and environmental stimuli were seen within both treatment groups (in-group differences in terms of change from baseline; $\bullet$ Table 2). While some statistically significant differences between SMART ${ }^{\mathrm{TM}}$-treated patients and the free-combination group were seen with regard to the mean AQLQ(S) global and domain scores (data not shown), the test used to generate the p-values did not take into account differences between the treatment groups in terms of the AQLQ(S) scores at baseline. As the SMARTTM group had higher AQLQ(S) scores at baseline, the change in the $\mathrm{AQLQ}(\mathrm{S})$ global score during the study was tested by ANCOVA with treatment as factor and baseline as covariate, to investigate the impact of these baseline differences. This test resulted in a p-value of 0.9545 , indicating no significant difference between the treatment groups in terms of the improvement in the global score of the AQLQ(S) when taking into consideration baseline $\mathrm{AQLQ}(\mathrm{S})$ scores.

\section{Health care utilization and asthma exacerbations}

Overall, the incidence of severe asthma exacerbations was similar in the SMART ${ }^{\mathrm{TM}}$ and free-combination treatment groups, both up to 3 months and between 3-6 months' follow-up ( $\bullet$ Table 3 ). This corresponds to an estimated mean annualised severe exacerbation rate of $0.20(95 \%$ CI $0.14,0.29)$ among SMART TM recipients and $0.17(95 \% \mathrm{Cl} 0.10,0.29)$ among those who received the

\begin{tabular}{|lllll|}
\hline & $\begin{array}{l}\text { SMART'M } \\
\text { (n=310) }\end{array}$ & & \multicolumn{2}{l|}{$\begin{array}{l}\text { Free combination } \\
(\mathbf{n = 1 7 2 )}\end{array}$} \\
\hline AQLQ(S) score, mean (SD) & Baseline & Change from baseline & Baseline & Change from baseline \\
\hline Global score & $5.37(1.02)$ & $0.25(0.82)$ & $4.89(1.19)$ & $0.42(0.89)$ \\
\hline Symptoms subscore & $5.34(1.12)$ & $0.23(0.97)$ & $4.84(1.26)$ & $0.41(1.01)$ \\
\hline Activity limitation subscore & $5.38(1.04)$ & $0.24(0.82)$ & $4.91(1.21)$ & $0.41(0.94)$ \\
\hline Emotional function subscore & $5.56(1.22)$ & $0.29(1.00)$ & $5.00(1.41)$ & $0.38(1.00)$ \\
\hline Environmental stimuli subscore & $5.20(1.31)$ & $0.24(1.01)$ & $4.84(1.43)$ & $0.46(1.17)$ \\
\hline
\end{tabular}

Table 2 Change from baseline to 6 months (study end) in AQLQ(S) scores in patients who received SMART TM or a free combination of ICS plus LABA and as-needed SABA (full analysis set; last observation carried forward) ${ }^{1}$.

Abbreviations: $A Q L Q(S)$, standardised version of the Asthma Quality of Life Questionnaire; ICS, inhaled corticosteroid; LABA, long-acting $\beta_{2}$-agonist; SABA, short-acting $\beta_{2}$-agonist; SD, standard deviation; SMARTTM ${ }^{\mathrm{M}}$, Symbicort ${ }^{\circledR}$ [budesonide/formoterol] maintenance and reliever therapy.

1 The differences between the two treatment groups in terms of the changes from baseline were evaluated by a t-test. While some of these differences attained statistical significance, the test does not take into account differences in baseline scores; the results of this testing are, therefore, not shown. 
Table 3 Severe asthma exacerbations in patients who received SMART TM or a free combination of ICS plus LABA and as-needed SABA (full analysis set).

\begin{tabular}{|c|c|c|}
\hline & $\begin{array}{l}\text { SMART }^{T M} \\
(n=310)\end{array}$ & $\begin{array}{l}\text { Free combination } \\
(n=172)\end{array}$ \\
\hline \multicolumn{3}{|l|}{ Exacerbations at $0-3$ months: } \\
\hline Patients, n (\%) & $10(3.2)$ & $5(2.9)$ \\
\hline Episodes per patient, range & $1-3$ & $1-2$ \\
\hline \multicolumn{3}{|l|}{ Exacerbations at $3-6$ months: } \\
\hline Patients, n (\%) & $13(4.2)$ & $8(4.6)$ \\
\hline Episodes per patient & 1 & 1 \\
\hline $\begin{array}{l}\text { Extrapolated mean annual } \\
\text { exacerbation rate, } n \text { episodes } \\
(95 \% \mathrm{Cl})\end{array}$ & $0.20(0.14,0.29)^{1}$ & $0.17(0.10,0.29)^{1}$ \\
\hline
\end{tabular}

The annual exacerbation rate was estimated by translating the reported numbers of episodes into estimated mean numbers per year using a Poisson regression model with treatment as factor and total time in study (in years) as offset variable.

Abbreviations: $\mathrm{Cl}$, confidence interval; ICS, inhaled corticosteroid; LABA, long-acting $\beta_{2}$-agonist; SABA, short-acting $\beta_{2}$-agonist; SMARTTM , Symbicort ${ }^{\otimes}$ [budesonide/formoterol] maintenance and reliever therapy.

${ }^{1}$ Between-treatment $\mathrm{p}=0.66$.

free combination. Throughout the study duration, two SMART ${ }^{\mathrm{TM}}$ recipients $(0.6 \%)$ required emergency room treatment and one patient in the free-combination group (0.6\%) was hospitalised due to asthma.

The time to the first severe asthma exacerbation and the time to first hospitalisation/emergency room treatment were not evaluated as no robust statistical comparisons could be made due to the small number of patients who experienced these endpoints.

\section{Lung function}

The baseline $\mathrm{FEV}_{1}$ values were similar between both groups (SMARTTM, 2.64 \pm 0.871 ; free combination $2.45 \pm 0.731$ [mean \pm $\mathrm{SD}])$. $\mathrm{FEV}_{1}$ improved in both groups during the study. The mean \pm SD improvement was slightly higher with SMART ${ }^{\mathrm{TM}}$ than in the free-combination group at both $3(0.06 \pm 0.33$ vs $0.03 \pm 0.331)$ and
$6(0.13 \pm 0.48$ vs $0.07 \pm 0.431)$ months (in-group differences were assessed, as per protocol, due to lack of randomisation).

\section{Daily inhaled steroid use}

The prescribed mean \pm SD daily dose of ICS (including rescue ICS) throughout the study duration was approximately $10 \%$ lower in the SMART ${ }^{\mathrm{TM}}$ group than in the free-combination group (615 \pm $318 \mu \mathrm{g}$ vs $678 \pm 380 \mu \mathrm{g}$, respectively).

\section{Use of systemic corticosteroids}

During the study, 13 (4.2\%) SMART ${ }^{\mathrm{TM}}$ and five (2.9\%) free-combination recipients used systemic corticosteroids.

\section{Treatment satisfaction}

High levels of treatment satisfaction were reported for both groups at baseline, although the proportion of patients who reported being very satisfied with their treatment was higher for SMART $^{\text {тм }}$ than for free combination treatment $(39.0 \%$ vs $23.3 \%$, respectively; Fig. 2). In both treatment arms, the number of patients who were either very or fairly satisfied with their asthma treatment increased slightly over time. At all timepoints, the proportion of patients who were very satisfied with treatment was higher (unadjusted for baseline) in the SMART'M group than among patients who received free-combination therapy. The proportion of patients who were very satisfied was $50.0 \%$ in the SMART ${ }^{\mathrm{TM}}$ group and $34.3 \%$ in the free-combination group at Visit 2, and $54.2 \%$ and $39.0 \%$, respectively, at Visit 3 ( Fig. 2). By Visit 3, $258(83.2 \%)$ patients in the SMART ${ }^{\mathrm{TM}}$ group and $120(69.8 \%)$ patients in the free-combination group confirmed a treatment satisfaction level of very satisfied or fairly satisfied.

\section{Healthcare resource utilisation and sick leave}

During the observation period, the proportion of patients who consulted a physician for any reason was similar with SMARTTM and free-combination therapy (105 [33.9\%] vs 62 [36.0\%] of patients, respectively). Additional diagnostic tests (e.g., pulmonary

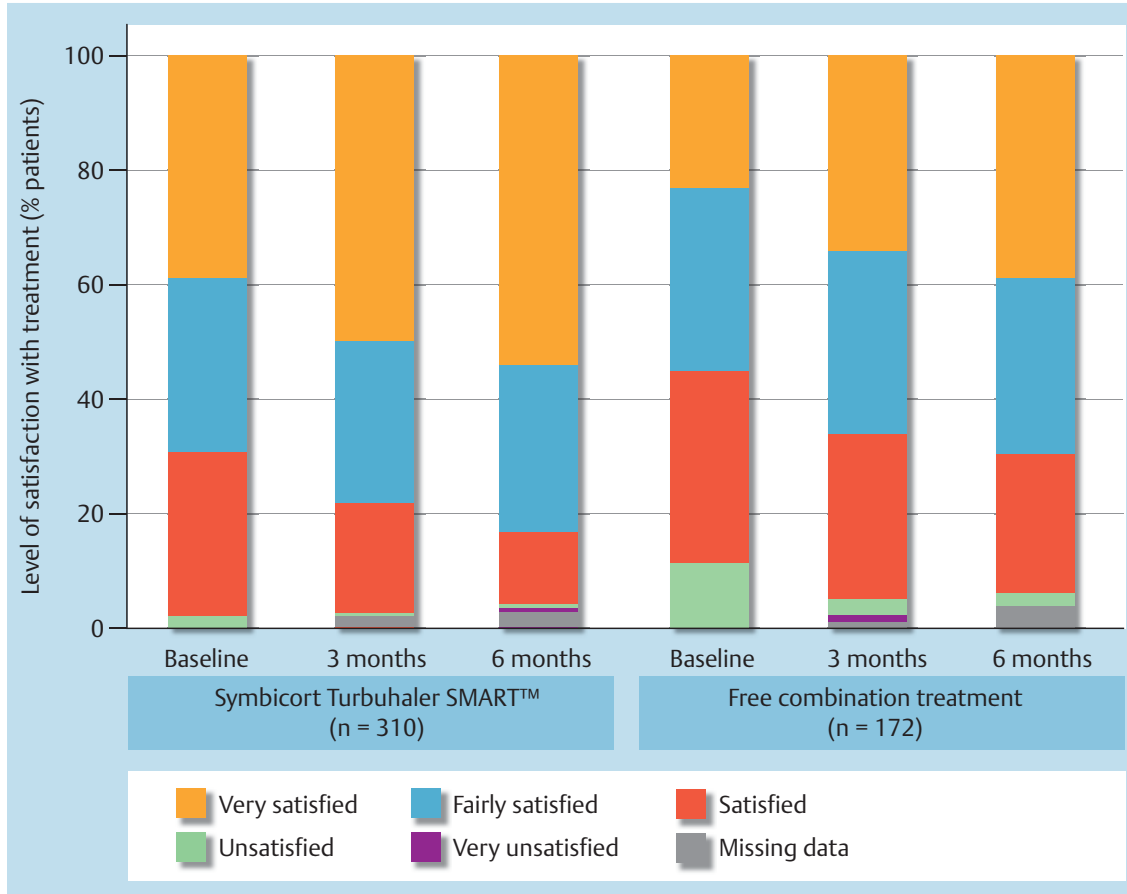

Fig. 2 Patients' level of satisfaction with SMARTTM or a free combination of ICS plus LABA and asneeded SABA at baseline, 3 months and 6 months (full analysis set). Abbreviations: ICS, inhaled corticosteroid; LABA, long-acting $\beta_{2}$-agonist; SABA, short-acting $\beta_{2}$-agonist; SMART, Symbicort ${ }^{\circledR}$ [budesonide/formoterol] maintenance and reliever therapy. 
function tests) were performed in $18(5.8 \%)$ patients in the SMART $^{\text {TM }}$ group and $16(9.3 \%)$ patients in the free-combination group.

Confirmed sick leave was reported for 27 (8.7\%) patients in the SMART $^{\mathrm{TM}}$ group and $11(6.4 \%)$ patients in the free-combination group.

\section{Safety}

Overall, seven $(2.2 \%)$ patients in the SMART ${ }^{\mathrm{TM}}$ group and seven (3.9\%) patients in the free-combination group reported one or more AEs. Three AEs were judged to be related to asthma treatment (one incidence of hoarseness and one of oral candidiasis in the free-combination group, and one case of hoarseness in the SMART $^{\mathrm{TM}}$ group). None of the serious AEs reported during the trial were considered to be related to either SMARTTM or freecombination therapy.

\section{Discussion}

$\nabla$

There is strong evidence of the benefits of SMART'M in the treatment of patients with asthma from the largest ever performed international asthma RCT program [5-7,9,12-16], and these findings have been supported by real-life studies $[9,17,18]$. However, all three of these real-life studies have assessed SMARTTM in randomised studies where the treatment arm was predefined. Data confirming the effectiveness of this treatment regimen in a reallife situation where all the patients are treated at the complete discretion of the physician are still sparse. This naturalistic noninterventional study, performed in a real-life clinical setting, therefore aimed to evaluate the use of rescue medication and HRQL in adult patients with a confirmed asthma diagnosis.

In this study, routine assessments were performed under real-life conditions, with no intervention by the sponsor with regard to patient selection, diagnostic procedures or therapeutic decisions. Thus, real-life patient management with no dose restrictions could be studied for both study arms (SMART ${ }^{\mathrm{TM}}$ and free combination) [19]. Real-life observations from non-interventional studies can be strikingly different from data gathered in RCTs. For example, recently published data show that leukotriene antagonists can be as effective as ICS in the routine asthma clinical setting, irrespective of a myriad of clinical trial data showing superiority of ICS [20]. Archibald Cochrane stated that "[b]etween measurements based on RCTs and benefit ... in the community there is a gulf which has been much under-estimated" [21].

Evaluation of asthma treatment success in patients in a real-life clinical situation is much more challenging than assessment of outcomes within the context of a RCT. The usual endpoints such as assessment of lung function at standardised control visits or physician assessment of exacerbations are not feasible in a noninterventional study, and daily measurements of peak expiratory flow would be too demanding for real-life conditions. Moreover, self-assessment of exacerbations is less reliable than physician assessment in RCTs. Thus, exacerbations could not be used as a primary outcome. Therefore, the number of rescue puffs per day was chosen as the most reliable, responsive and ascertainable single primary variable for assessment of asthma control. The choice of this parameter as the primary outcome is supported by a recent publication, which showed that airway obstruction lability may allow for a more complete assessment of disease activity [22]. Furthermore, this endpoint also allows evaluation of dropouts without introducing a 'healthy survivor' bias. Evaluations of
HRQL and other conventional asthma-related efficacy parameters were chosen as secondary variables in this study.

In our setting, a sample size with 2:1 allocation (SMART ${ }^{\mathrm{TM}}$ and free combination) was calculated assuming a between-treatment difference of 0.506 puffs of rescue medication per day, based on data attained from a previous RCT (DESOLO), which had a very similar design [11]. In the 498 patients enrolled in the present study, the least squares mean for the difference between treatment groups was only one-half of this estimate $(-0.266 ; \mathrm{p}=$ $0.013)$. The smaller between-treatment difference may have been caused by the smaller mean daily number of rescue medication puffs than expected based on data from DESOLO, and further highlights the difference between pragmatic non-interventional studies and RCTs. Nevertheless, a significant benefit of SMART ${ }^{\text {TM }}$ can be shown for the primary outcome.

Shortly after completion of the EuroSMART RCT [9], which had similar inclusion criteria to those for the present non-interventional study, the same investigators were recruited. Consequently, the EuroSMART cohort and the patient population included in the present study are comparable. Prior to inclusion, $>88 \%$ of patients in both treatment arms had been receiving the same asthma treatment for $>6$ months. This resulted in a population of patients who had good HRQL and high levels of satisfaction with treatment in both groups. We therefore may have observed a ceiling effect for further clinically meaningful changes, consecutively seeing a small effect, even in cases where the recorded changes achieved statistical significance. It is also important to note that the $\operatorname{AQLQ}(S)$ endpoint comparison is biased due to baseline differences since the study was not randomised). While the mean $A Q L Q(S)$ global score was slightly higher in the SMART $^{\mathrm{TM}}$ group than in the free-combination group at study end, this value at baseline was considerably higher in the SMART $^{\mathrm{TM}}$ vs the free-combination treatment group (with the resulting difference being greater in the free-combination group). When baseline AQLQ(S) score was included in the statistical analysis there was no difference between the treatment groups.

Similarly, the mean annualised number of exacerbations in the present study was low ( 0.20 episodes for SMART ${ }^{\text {TM }}$ vs 0.17 episodes for free-combination treatment). Again, this incidence is less than one-half of the expected, given that patients who had at least one severe exacerbation in the previous 24 months were recruited. However, this has previously been observed in large RCTs and could be partially related to the precision of retrospectively collected data. Regardless of the cause of this discrepancy, asthma exacerbations requiring systemic corticosteroids were rare in this population of intensively pre-treated patients who were satisfied with their treatment and had good HRQL. Therefore, robust statistical analysis of the difference between treatment groups could not be performed. The reasons outlined above, together with the difference in exacerbation reporting between RCTs and this real-life study, would also explain the lack of difference between treatments in the reduction in number of exacerbations observed here compared with the greater reduction in number of exacerbations observed with SMART ${ }^{\mathrm{TM}}$ vs comparator arms in RCTs [23].

As expected, clinic assessments of $\mathrm{FEV}_{1}$, with no standardised washout periods as is the case in real life, are not suitable for evaluation of between-group differences. There was, however, a marked mean improvement from baseline, especially in the SMART $^{\text {TM }}$ group, despite the fact that this population had better $\mathrm{FEV}_{1}$ values at baseline. This could also be related to the better adherence observed with treatment with just one inhaler. 
If the number of rescue medication puffs per day, HRQL and the number of exacerbations are a surrogate marker for asthma control, it is notable that the same, or better, control can be achieved with a lower corticosteroid burden, despite the fact that ICS was also used (in addition to LABA) in the SMART ${ }^{\mathrm{TM}}$ group as a reliever medication, as well as part of maintenance treatment.

The strengths of this non-interventional study include close quality assurance and on-site monitoring, experienced investigators who participated in the previous EuroSMART RCT [9] and verified asthma diagnosis, resulting in a relatively homogenous population with GINA Step 3 treatment and presumed severity grade of 3 or 4 . This is particularly important given prior findings that one-third of patients treated with asthma drugs in the community do not have asthma [24]. The real-life setting of this study may be considered a bias; however, we consider this to be a strength, as it means that we were able to clarify the effectiveness of SMART TM outside the stringent RCT environment.

Limitations inherent to the nature of the non-interventional study design should be considered. Real-life patient adherence is not comparable with surveillance in RCTs for a number of reasons including drug holidays, running out of drugs and drug preferences, among many. Indeed, register studies of 1-year treatment duration have shown that patients take far less than onehalf of their prescribed doses [25-27]. The most important limitation of this non-interventional study is the fact that patients who previously took part in a RCT were included. As already discussed, these patients could have been better controlled and more adequately pre-treated in comparison with treatment-naïve or non-study patients. A confounding factor to this is that the prescribed doses used in the free-combination arm of the study were at the discretion of the treating physician according to the recommendations of the package inserts of each medication; this could result in possible bias and create a wide variety of ICS/LABA treatment regimens (and possibly outcomes) for the free-combination arm. Despite this, benefits in the daily number of rescue medication puffs used (primary endpoint) were observed.

Most secondary variables showed either significant or numerical benefit in favour of SMART ${ }^{\mathrm{TM}}$ or the two treatment regimens were, for other secondary variables, at least equally effective. The differences between the treatment groups that were observed in this non-interventional study are not as marked as those previously seen in the SMART RCTs, particularly with regard to HRQL $[6,18]$. A number of factors may underlie these blunted effect sizes. Firstly, RCTs select the 'best candidates' for treatment; i.e., pre-treated, but still symptomatic, patients who have room for improvement $[5-7,9,12-16,18]$. Non-interventional studies offer treatments for patients who are less likely to show improvements, such as the pre-treated population enrolled in the present study. Secondly, real-life patient assessment is inferior to RCT patient assessment, as RCTs can enforce a standardised treatment duration and washout periods, as well as being able to assess trough and peak effects. In this study, we aimed to assess real-life management of patients with asthma and the treating physicians were given limited guidance regarding therapeutic decisions. Thirdly, in real-life naturalistic trials, patients with significant co-morbidities and concomitant treatments with potential interactions are included. While the physicians were asked to consider the prescribing information for SMART ${ }^{\mathrm{TM}}$ and the medications used in the free combination plus as-needed SABA therapy arm, we cannot guarantee that patients did not receive contraindicated concomitant medications.
Treatment adherence with the simple SMART ${ }^{\mathrm{TM}}$ strategy could play an important role compared with conventional three inhaler treatment; however, the non-interventional study design is not suitable for assessment of adherence.

\section{Conclusion}

In this real-life setting, patients treated with SMART ${ }^{\mathrm{TM}}$ needed less rescue medication despite a lower inhaled corticosteroid burden when compared with conventional asthma management with three separate inhaler devices.

\section{Conflict of Interest \\ $\nabla$}

P. Kardos was reimbursed by AstraZeneca for his participation in designing the study, discussing the evaluation and preparing the conception for the publication; moreover, his institution participated in the study as a centre. In the last 5 years, PK has received fees for consultation and presentations, or was reimbursed for travel expenses, from the following companies: Boehringer Ingelheim, Chiesi, GlaxoSmithKline, Merck Sharp \& Dohme, Mundipharma, Novartis and Talecris. Editorial assistance was funded by AstraZeneca.

\section{Acknowledgements}

$\nabla$

The authors thank Anna Mett of inScience Communications, Springer Healthcare, for editorial assistance.

\section{References}

1 Global Initiative for Asthma. Global strategy for asthma management and prevention: updated 2010. 2010: Available from: http://www.ginasthma.org/pdf/GINA_Report_2010.pdf [04 Jan 2012]

2 Scottish Intercollegiate Guidelines Network/British Thoracic Society. British guideline on the management of asthma: A national clinical guideline: revised. 2011: Available from: http://www.brit-thoracic. org.uk/Portals/0/Guidelines/AsthmaGuidelines/sign101\%20Sept\% 202011.pdf [05 Jan 2012]

3 Chowdhury BA, Dal Pan G. The FDA and safe use of long-acting betaagonists in the treatment of asthma. N Engl J Med 2010; 362: 11691171

4 AstraZeneca. Symbicort Turbohaler 200/6 Inhalation powder: Summary of product characteristics. 2011: Available from: http://www.medicines.org.uk/emc/medicine/4821/SPC/ [23 Jan 2012]

5 Bousquet J, Boulet LP, Peters MJ et al. Budesonide/formoterol for maintenance and relief in uncontrolled asthma vs. high-dose salmeterol/ fluticasone. Respir Med 2007; 101: 2437-2446

6 Kuna P, Peters MJ, Manjra AI et al. Effect of budesonide/formoterol maintenance and reliever therapy on asthma exacerbations. Int J Clin Pract 2007; 61: $725-736$

7 O'Byrne PM, Bisgaard H, Godard PP et al. Budesonide/formoterol combination therapy as both maintenance and reliever medication in asthma. Am J Respir Crit Care Med 2005; 171: 129-136

8 Travers J, Marsh S, Williams $M$ et al. External validity of randomised controlled trials in asthma: to whom do the results of the trials apply? Thorax 2007; 62: 219-223

9 Aubier M, Buhl R, Ekstrom T et al. Comparison of two twice-daily doses of budesonide/formoterol maintenance and reliever therapy. Eur Respir J 2010; 36: 524-530

10 Juniper EF, Buist AS, Cox FM et al. Validation of a standardized version of the Asthma Quality of Life Questionnaire. Chest 1999; 115: 12651270 
11 AstraZeneca. A comparison of Symbicort Single inhaler Therapy (Symbicort Turbuhaler 160/4.5 $\mu \mathrm{g}, 1$ inhalation b.i.d. plus as needed) and conventional best practice for the treatment of persistent asthma in adults - a 26-week, randomised, open-label, parallel-group, multicentre study. 2009: Available from: http://www.astrazenecaclinicaltrials. com/_mshost800325/content/clinical-trials/resources/pdf/ D5890L00011 [27 Feb 2012]

12 Rabe KF, Atienza T, Magyar P et al. Effect of budesonide in combination with formoterol for reliever therapy in asthma exacerbations: a randomised controlled, double-blind study. Lancet 2006; 368: 744-753

13 Rabe KF, Pizzichini E, Stallberg B et al. Budesonide/formoterol in a single inhaler for maintenance and relief in mild-to-moderate asthma: a randomized, double-blind trial. Chest 2006; 129: 246-256

14 Scicchitano $R$, Aalbers $R$, Ukena $D$ et al. Efficacy and safety of budesonide/formoterol single inhaler therapy versus a higher dose of budesonide in moderate to severe asthma. Curr Med Res Opin 2004; 20 : $1403-1418$

15 Louis R, Joos G, Michils A et al. A comparison of budesonide/formoterol maintenance and reliever therapy vs. conventional best practice in asthma management. Int J Clin Pract 2009; 63: 1479-1488

16 Lundborg M, Wille S, Bjermer L et al. Maintenance plus reliever budesonide/formoterol compared with a higher maintenance dose of budesonide/formoterol plus formoterol as reliever in asthma: an efficacy and cost-effectiveness study. Curr Med Res Opin 2006; 22: 809-821

17 Demoly P, Louis R, Soes-Petersen $U$ et al. Budesonide/formoterol maintenance and reliever therapy versus conventional best practice. Respir Med 2009; 103: 1623-1632

18 Vogelmeier C, D'Urzo A, Pauwels $R$ et al. Budesonide/formoterol maintenance and reliever therapy: an effective asthma treatment option? Eur Respir J 2005; 26: 819-828
19 Godwin M, Ruhland L, Casson I et al. Pragmatic controlled clinical trials in primary care: the struggle between external and internal validity. BMC Med Res Methodol 2003; 3: 28

20 Price $D$, Musgrave $S D$, Shepstone $L$ et al. Leukotriene antagonists as firstline or add-on asthma-controller therapy. N Engl J Med 2011; 364: $1695-1707$

21 Cochrane AL. Effectiveness and Efficiency: random reflections on Health Services. London: Nuffield Provincial Hospitals Trust; 1972

22 Greenberg S, Liu N, Kaur A et al. Airway obstruction lability helps distinguish levels of disease activity in asthma. Respir Med 2012; 106: $500-507$

23 Cates CJ, Karner C. Combination formoterol and budesonide as maintenance and reliever therapy versus current best practice (including inhaled steroid maintenance), for chronic asthma in adults and children. Cochrane Database Syst Rev 2013; 4: CD007313

24 Aaron SD, Vandemheen KL, Boulet LP et al. Overdiagnosis of asthma in obese and nonobese adults. Can Med Ass J 2008; 179: 1121 - 1131

25 Stempel DA, Stoloff SW, Carranza Rosenzweig JR et al. Adherence to asthma controller medication regimens. Respir Med 2005; 99: 1263 1267

26 Marceau C, Lemiere C, Berbiche D et al. Persistence, adherence, and effectiveness of combination therapy among adult patients with asthma. J Allergy Clin Immunol 2006; 118: 574-581

27 Stoloff SW, Stempel DA, Meyer J et al. Improved refill persistence with fluticasone propionate and salmeterol in a single inhaler compared with other controller therapies. J Allergy Clin Immunol 2004; 113: $245-251$ 